\title{
Erratum: First-principles DFT+DMFT calculations of structural properties of actinides: Role of Hund's exchange, spin-orbit coupling, and crystal structure [Phys. Rev. B 94, 115148 (2016)]
}

\author{
Bernard Amadon
}

(Received 22 November 2017; published 25 January 2018)

DOI: 10.1103/PhysRevB.97.039903

The calculations reported in the original paper contain an error: The effective Hund's coupling $J$ used in the combination of density functional theory and dynamical mean field theory (DFT+DMFT) does not use the same convention as the effective Hund's coupling $J$ computed in the constrained random-phase approximation (cRPA). The definition of $J$ presented in Ref. [1] and used in the original paper in the cRPA calculation follows the expression of $J_{1}$ :

$$
J_{1}=\frac{1}{4} \sum_{\sigma, \sigma^{\prime}} \frac{1}{(2 l+1)(2 l)} \sum_{m_{1}=1}^{2 l+1} \sum_{m_{2}=1\left(m_{2} \neq m_{1}\right)}^{2 l+1} U_{m_{1}, m_{2}, m_{2}, m_{1}}^{\sigma, \sigma^{\prime}} .
$$

A more common definition of $J$ can however be established using the expression of $J_{2}$ deduced from (see Ref. [2] or the Appendix of Ref. [3])

$$
U-J_{2}=\frac{1}{4} \sum_{\sigma, \sigma^{\prime}} \frac{1}{(2 l+1)(2 l)} \sum_{m_{1}=1}^{2 l+1} \sum_{m_{2}=1}^{2 l+1}\left(U_{m_{1}, m_{2}, m_{1}, m_{2}}^{\sigma, \sigma^{\prime}}-U_{m_{1}, m_{2}, m_{2}, m_{1}}^{\sigma, \sigma^{\prime}}\right) .
$$

As discussed in Ref. [4], even if the second expression is used more, the choice between Eqs. (1) and (2) is a matter of convention. However, for self-consistent calculations as those used in the original paper, there is an error because the convention used for $J$ in DFT $+U$ in the ABINIT code [5] is the one from Eq. (2) and not the one from Eq. (1). Moreover, in the original paper, the $J$ values computed with Eq. (1) were used in a DFT+DMFT code which uses Eq. (2).

So, first, we have reproduced the self-consistent cRPA calculations of effective interactions. They are reproduced in Table I and Fig. 2 for actinides. The (correct) values of $J$, defined by Eq. (2), are around $0.1 \mathrm{eV}$ larger. The values of $U$ are not affected.

As the DFT+DMFT calculations use these values of $U$ and $J$, we also recompute all the data for all figures that depend on $J$ (see Tables II, III, Figs. 3, 4, and 6-9).

Importantly, we see in Fig. 3 and Table II that the quantitative agreement of the DFT+DMFT calculations with experiments is improved with the correct value of the Hund's interaction. The interpretations and discussions of all figures and tables are unchanged, except that the role of $J$ is larger (see Fig. 4).

TABLE I. The values in $\mathrm{eV}$ of the effective and bare interactions for actinides. $U^{\text {crpa }}$ is the $F^{0}$ Slater integral, and $U_{\text {diag }}^{\text {crpa }}$ is the average of the diagonal elements of the direct Coulomb interaction matrix (see, e.g., Refs. [1,4]).

\begin{tabular}{lrrrrr}
\hline \hline & $\mathrm{U}$ & $\mathrm{Np}$ & $\mathrm{Pu}$ & $\mathrm{Am}$ & $\mathrm{Cm}$ \\
\hline$U^{\text {crpa }}$ & 0.8 & 1.0 & 0.95 & 1.5 & 3.4 \\
$U_{\text {diag }}^{\text {crpa }}$ & 1.5 & 1.7 & 1.7 & 2.3 & 4.3 \\
$J^{\text {crpa }}$ & 0.5 & 0.5 & 0.6 & 0.5 & 0.7 \\
$U^{\text {bare }}$ & 17.6 & 18.4 & 18.6 & 0.0 & 0.7 \\
$J^{\text {bare }}$ & 0.7 & 0.7 & 0.8 & 0.8 \\
\hline \hline
\end{tabular}




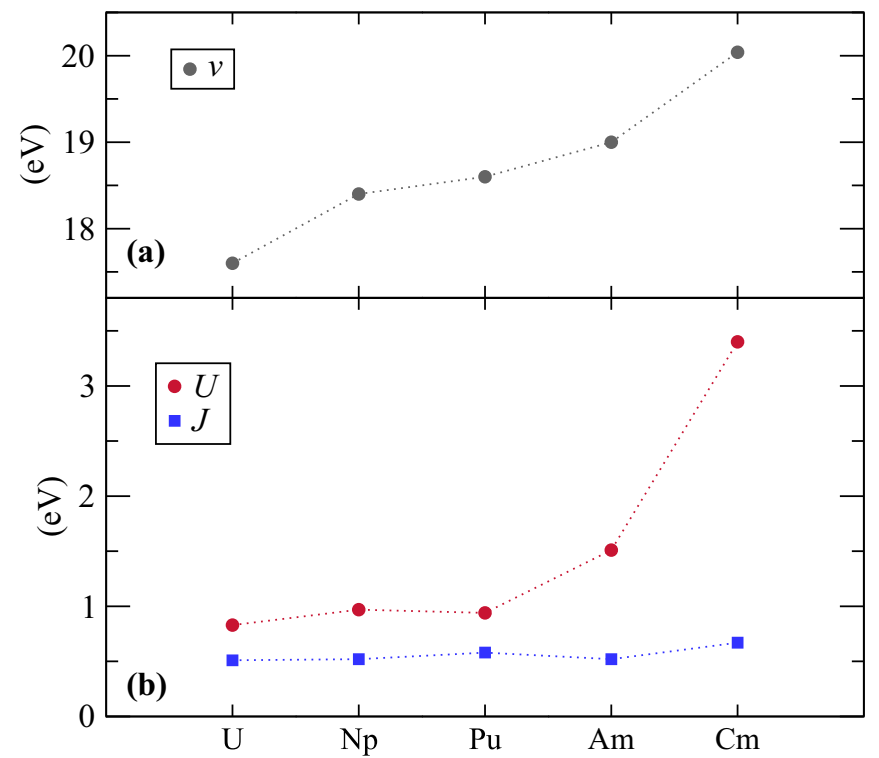

FIG. 2. Ab initio bare and effective interactions in actinides. Bare direct Coulomb interaction $v$ [in part (a)], the Hubbard $U$, and the Hund's exchange $J$ [in part (b)] interactions for $\mathrm{U}, \mathrm{Np}, \mathrm{Pu}, \mathrm{Am}$, and $\mathrm{Cm}$.

TABLE II. Structural parameters of uranium, neptunium, americium, curium, and some phases of plutonium obtained in this paper in comparison with various other methods and experiment. NM, AFM, and OP stand for nonmagnetic calculation, antiferromagnetic calculation, and orbital polarization (see Sec. IV of the original paper). See also the Supplemental Material of the original paper, Sec. I for a benchmark of our NM GGA calculations with other calculations.

\begin{tabular}{|c|c|c|c|c|c|c|c|c|c|}
\hline & $\alpha-\mathrm{U}$ & $\alpha-\mathrm{Np}$ & $\widetilde{\alpha}-\mathrm{Pu}$ & $\alpha-\mathrm{Pu}$ & $\delta-\mathrm{Pu}$ & $\gamma-\mathrm{Pu}$ & $\epsilon-\mathrm{Pu}$ & Am & $\mathrm{Cm}$ \\
\hline \multicolumn{10}{|c|}{ Experiment $[11,13,15,16,18,19]$} \\
\hline$V\left(\AA^{3}\right)$ & $20.6[11]$ & $19.2[20]$ & & $20.08[13]$ & $25.0[19]$ & $23.5[19]$ & 24.4 [19] & $29.3[16]$ & $30.05[18]$ \\
\hline$B_{0}(\mathrm{GPa})$ & $115[11]$ & $120[21]$ & & 54 [13] & 29 to 30 [19] & $25.7[19]$ & 45 [19] & $29.7[16]$ & $30.5[18]$ \\
\hline \multicolumn{10}{|c|}{ Experiment extrapolated to $0 \mathrm{~K}$ (Lanata et al. [6] and Wallace [22]) } \\
\hline$V\left(\AA^{3}\right)$ & & & & 19.5 & 25.5 & 21.9 & 22.3 & & \\
\hline$B_{0}(\mathrm{GPa})$ & & & & $70[14]$ & 38 & & & & \\
\hline \multicolumn{10}{|c|}{ GGA/SOC+DMFT (NM) (this paper) } \\
\hline$V\left(\AA^{3}\right)$ & 20.5 & 18.9 & 19.2 & & 25.2 & 21.6 & 22.5 & 29.1 & 28.5 \\
\hline$B_{0}(\mathrm{GPa})$ & 124 & 143 & 103 & & 34 & 42 & 17 & 35 & 34 \\
\hline$E(\mathrm{meV})$ & & & 0 & & 108 & 39 & 126 & & \\
\hline \multicolumn{10}{|c|}{ GGA/SOC (NM) (this paper) } \\
\hline$V\left(\AA^{3}\right)$ & 20.2 & 18.5 & 18.0 & 17.8 & 19.5 & 17.9 & 17.2 & 19.9 & 20.8 \\
\hline$B_{0}(\mathrm{GPa})$ & 139 & 174 & 193 & 141 & 86 & 124 & 142 & 61 & 54 \\
\hline$E(\mathrm{meV})$ & & & 0 & -45 & 700 & 400 & 380 & & \\
\hline \multicolumn{10}{|c|}{$\mathrm{LDA} / \mathrm{SOC}+\mathrm{GA}(\mathrm{NM})[6] 0 \mathrm{~K}(U=4.5 \mathrm{eV}, J=0.36 \mathrm{eV})$} \\
\hline$V\left(\AA^{3}\right)$ & & & & 21.1 & 25.5 & 21.3 & 21.2 & & \\
\hline$B_{0}(\mathrm{GPa})$ & & & & $50-70$ & $15-35$ & $45-70$ & $35-55$ & & \\
\hline$E(\mathrm{meV})$ & & & & 0 & 26 & 9 & 72 & & \\
\hline GGA & $\mathrm{NM}$ & NM & & AFM & AFM & AFM & AFM & AFM & AFM \\
\hline$V\left(\AA^{3}\right)$ & $20.2[11]$ & $18.4-18.8[12,23]$ & & 18.5 [7] & 23.4 [7] & 21.9 [7] & $21.2[7]$ & $29.2[8]$ & $29.34[8]$ \\
\hline$B_{0}(\mathrm{GPa})$ & $134[11]$ & $158-170[12,23]$ & & $101[7]$ & $55[7]$ & $45[7]$ & 45 [7] & $28.3[8]$ & $40.3[8]$ \\
\hline$E(\mathrm{meV})$ & & & & 0 [7] & $122[7]$ & 109 [7] & $175[7]$ & & \\
\hline \multicolumn{10}{|c|}{ GGA/SOC+OP (AFM) $[9,10]$} \\
\hline$V\left(\AA^{3}\right)$ & & & & 20.3 & 24.9 & 24.3 & 24.6 & 29 & 28.5 \\
\hline$B_{0}(\mathrm{GPa})$ & & & & 50 & 41 & 33 & 23 & & \\
\hline$E(\mathrm{meV})$ & & & & 0 & 20 & 15 & 110 & & \\
\hline
\end{tabular}


TABLE III. Comparison of our DFT+DMFT calculations (*) of volumes and bulk moduli with other DFT+DMFT calculations and DFT+GA calculations. For completeness, we show the structural data obtained with our $a b$ initio interactions and with the interactions proposed by some authors. IS stands for interpolation scheme (see Ref. [24]).

\begin{tabular}{|c|c|c|c|}
\hline & $U, J(\mathrm{eV})$ & $V\left(\AA^{3}\right)$ & $B_{0}(\mathrm{GPa})$ \\
\hline \multicolumn{4}{|l|}{$\delta$-plutonium } \\
\hline GGA + DMFT/CTQMC* (continuous-time quantum Monte Carlo) & $0.94,0.58$ & 25.2 & 34 \\
\hline $\mathrm{LDA}+\mathrm{GA}[6]$ & $4.50,0.36$ & 25.5 & $15-35$ \\
\hline $\mathrm{LDA}+\mathrm{DMFT} / \mathrm{CTQMC} *$ & $4.50,0.36$ & 24.3 & 43 \\
\hline GGA + DMFT/IS [24] & $4.00,0.00$ & 26.5 & \\
\hline $\mathrm{GGA}+\mathrm{DMFT} / \mathrm{CTQMC} *$ & $4.00,0.00$ & 25.8 & 28 \\
\hline \multicolumn{4}{|l|}{ Americium } \\
\hline GGA + DMFT/CTQMC* & $1.53,0.52$ & 29.1 & 35 \\
\hline LDA + DMFT/Hubbard I [25] & $4.50,0.60$ & 27.4 & 45 \\
\hline $\mathrm{LDA}+\mathrm{DMFT} / \mathrm{CTQMC}{ }^{*}$ & $4.50,0.60$ & 28.0 & 44 \\
\hline
\end{tabular}

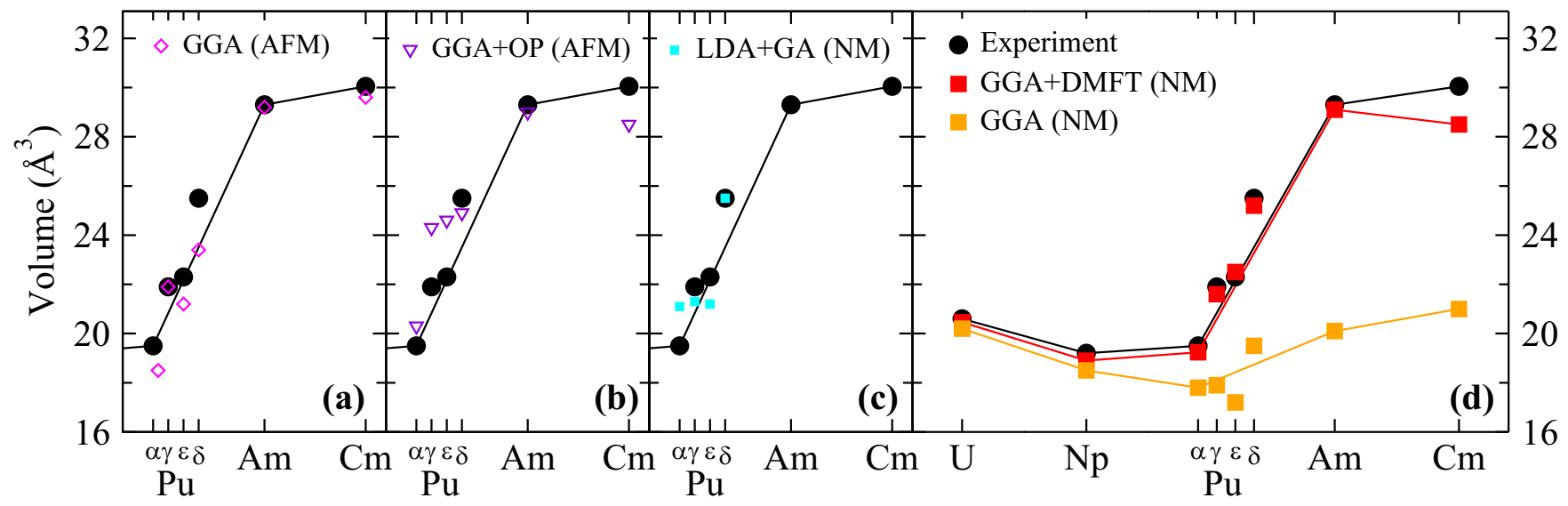

FIG. 3. Theoretical volumes for actinides compared to experiment. (d) Theoretical volumes as obtained in the DFT/PBE (Perdew-BurkeErnzerhof) (NM) and in the DFT/PBE+DMFT - with the calculated values of the effective direct Coulomb and Hund's interactions (see Fig. 2) in comparison to experiment (extrapolated to $0 \mathrm{~K}$ for $\gamma, \epsilon$, and $\delta \mathrm{Pu}$ [6]), (a) generalized gradient approximation (GGA) (AFM) [7,8], (b) GGA+OP (AFM) [9,10], and (c) local-density approximation (LDA)+GA (NM) [6]. Experimental data are taken from Refs. [11-18]. NM refers to a nonmagnetic calculation without any local or global static magnetic moment. AFM refers to antiferromagnetic calculations. GGA+DMFT with the $a b$ initio determination of $U$ and $J$ is able to describe the equilibrium volumes of all elements and all phases of Pu without any magnetic moment, in agreement with experiment. See Sec. IV of the original paper for a detailed discussion.

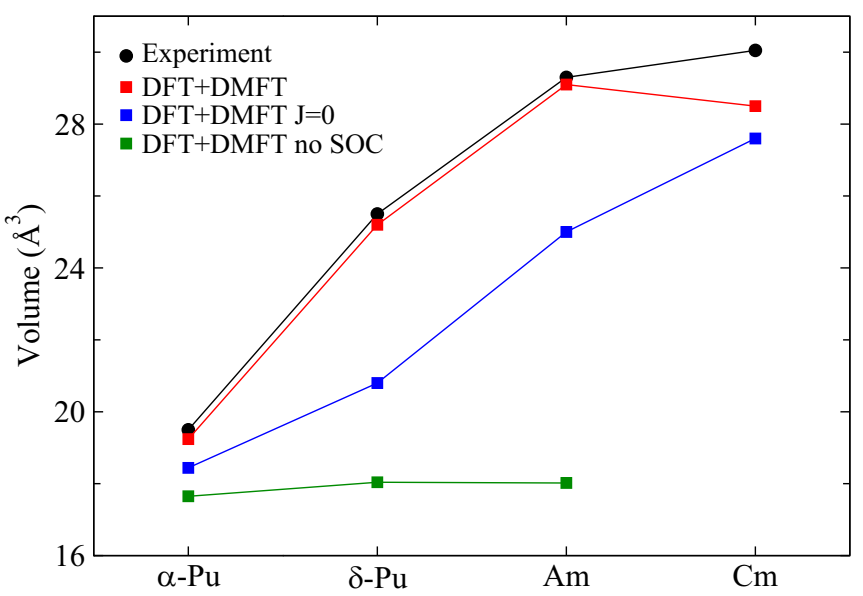

FIG. 4. The role of spin-orbit coupling (SOC) and exchange. Volumes (per atom) of $\alpha$-Pu, $\delta$-Pu, and Am for the full DMFT calculations (with $J$ and SOC) for calculations without exchange $J$ and for calculations without SOC, in comparison to experiment. The nonmagnetic calculation for curium is not stable without spin-orbit coupling. These calculations show that the combination of both spin-orbit coupling and exchange $J$ is necessary to recover the equilibrium volume of $\delta$-Pu and Am. 


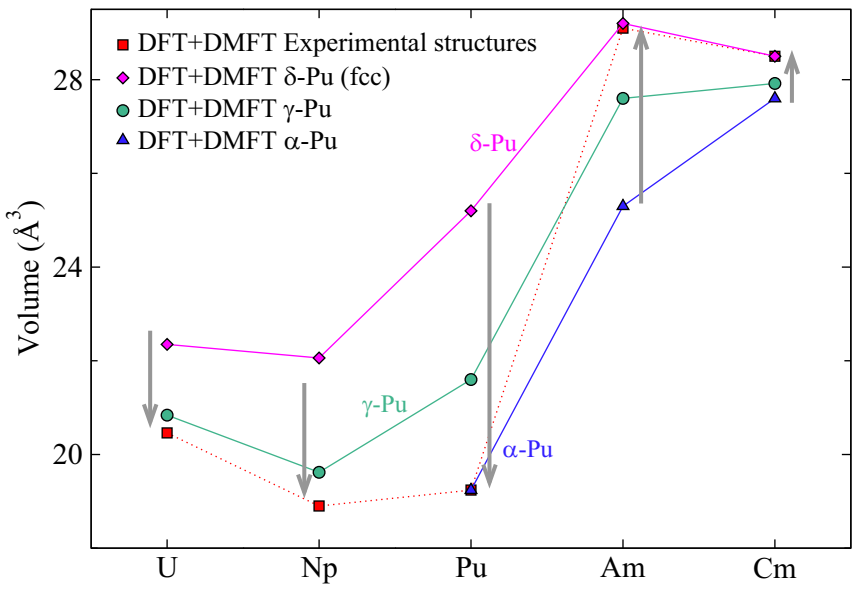

FIG. 6. The role of the crystal structure on theoretical volumes. Comparison of DFT+DMFT calculations of volumes (per atom) for actinides with fcc structures, the $\gamma$-Pu structure, the $\delta$-Pu structure, and experimental structures. The arrows show the direction of the decrease in DFT+DMFT energy towards the most stable phase (in agreement with experiment). For, e.g., plutonium, we indeed found that the $\alpha$ phase is more stable than the $\gamma$ phase whereas the $\delta$-fcc phase is less stable, in agreement with experiment.

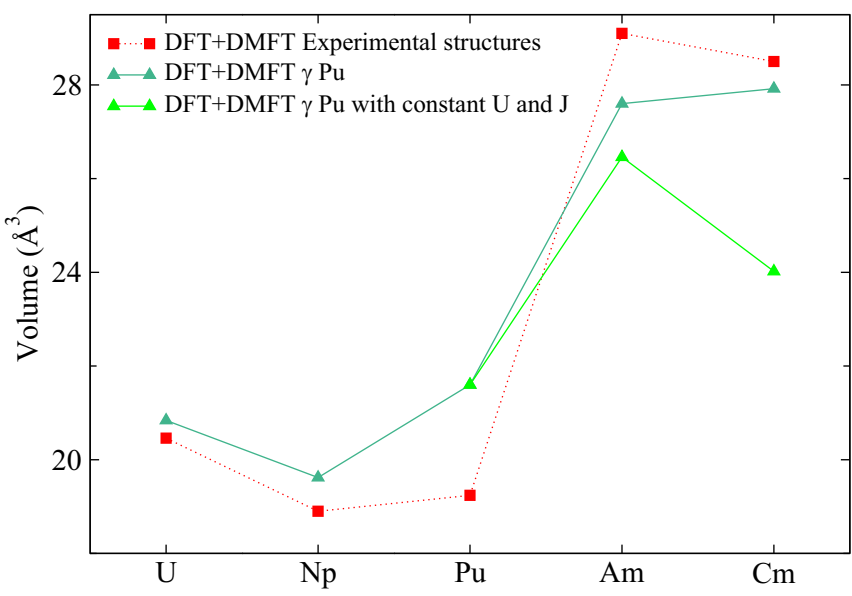

FIG. 7. DFT+DMFT calculations of volumes (per atom) for actinides with the same imposed structure (the $\gamma$ phase of plutonium). The graph compares calculations with computed $U$ and $J$ for each actinide with calculations with fixed $U$ and $J$. For reference, DFT + DMFT calculations for experimental structures also are given.

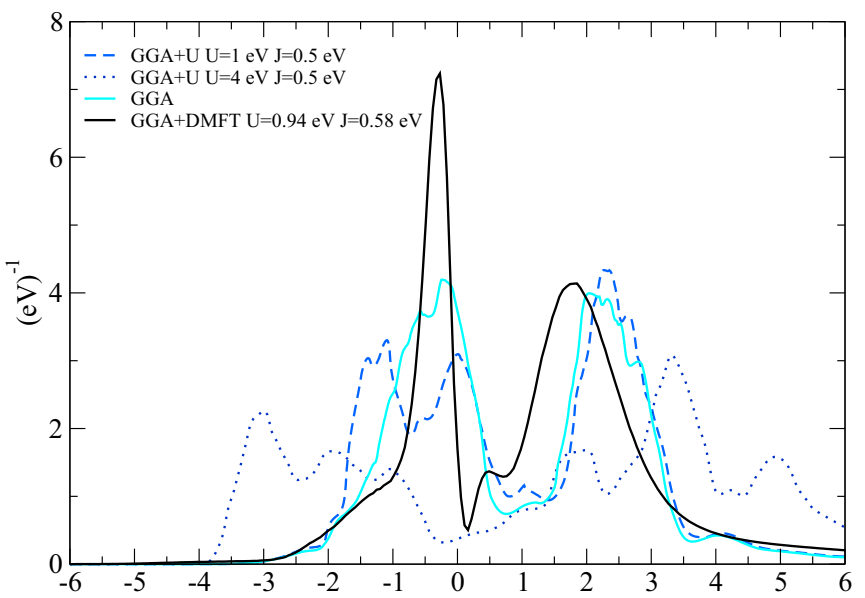

FIG. 8. DFT+DMFT $5 f$ spectral functions of $\delta$-Pu compared to the GGA and GGA $+U 5 f$ projected density of states (DOS) used for the cRPA calculation of $U$. Calculations are performed at $800 \mathrm{~K}$ and GGA $+U$, and GGA DOS are widened (with a 0.5 -eV broadening) to be compared easily to the DFT+DMFT spectral function. 

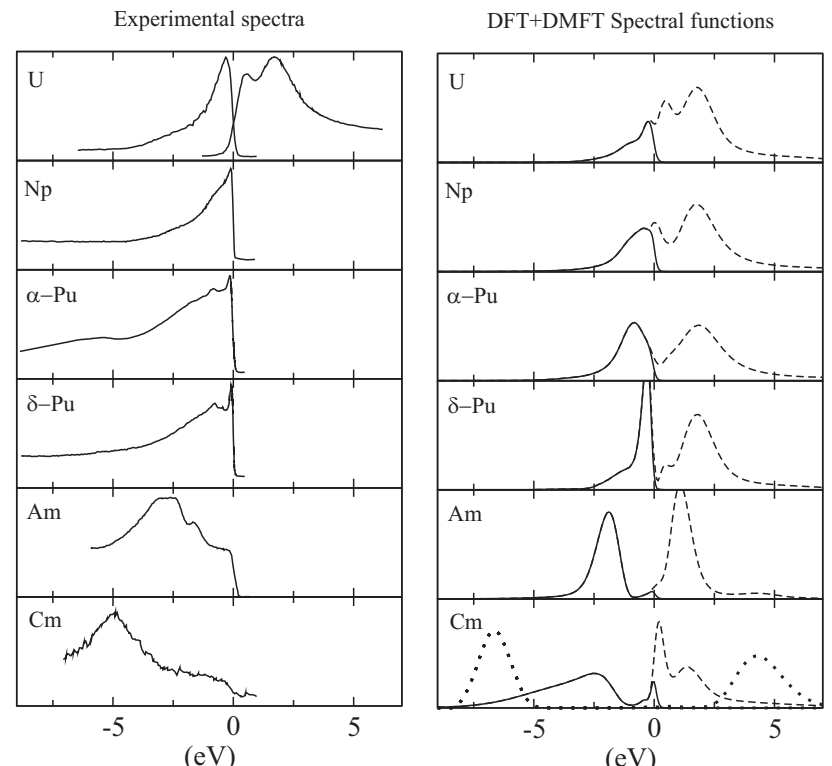

FIG. 9. Experimental photoemission spectra (left) of $\alpha$ uranium (398 K) [26], $\alpha$ neptunium (80 K) [27], plutonium ( $\alpha$ at $423 \mathrm{~K}$ and $\delta$ at $77 \mathrm{~K}$ ) [28], americium (at $298 \mathrm{~K}$ ) [29], and curium (298 K) [30], compared to our DFT+DMFT spectral functions (right) computed with $a b$ initio effective interactions computed with the self-consistent cRPA scheme. The full spectral function and theoretical direct photoemission spectra are presented with the dashed and full curves, respectively. The results are nearly identical at 800 and $200 \mathrm{~K}$. The magnetic calculations for curium are indicated by the dotted lines.

[1] B. Amadon, T. Applencourt, and F. Bruneval, Screened Coulomb interaction calculations: cRPA implementation and applications to dynamical screening and self-consistency in uranium dioxide and cerium, Phys. Rev. B 89, 125110 (2014).

[2] V. I. Anisimov, I. V. Solovyev, M. A. Korotin, M. T. Czyzyk, and G. A. Sawatzky, Density-functional theory and NiO photoemission spectra, Phys. Rev. B 48, 16929 (1993).

[3] M. T. Czyzyk and G. A. Sawatzky, Local-density functional and on-site correlations: The electronic structure of $\mathrm{La}_{2} \mathrm{CuO}_{4}$ and $\mathrm{LaCuO}_{3}$, Phys. Rev. B 49, 14211 (1994).

[4] B. Amadon, T. Applencourt, and F. Bruneval, Erratum: Screened Coulomb interaction calculations: cRPA implementation and applications to dynamical screening and self-consistency in uranium dioxide and cerium [Phys. Rev. B 89, 125110 (2014)], Phys. Rev. B 96, 199907 (2017).

[5] B. Amadon, F. Jollet, and M. Torrent, $\gamma$ and $\beta$ cerium: LDA $+U$ calculations of ground-state parameters, Phys. Rev. B 77, 155104 (2008).

[6] N. Lanatà, Y. Yao, C.-Z. Wang, K.-M. Ho, and G. Kotliar, Phase Diagram and Electronic Structure of Praseodymium and Plutonium, Phys. Rev. X 5, 011008 (2015).

[7] G. Robert, A. Pasturel, and B. Siberchicot, Calculated thermodynamic properties of plutonium metal, J. Phys.: Condens. Matter 15, 8377 (2003).

[8] A. Kutepov and S. Kutepova, First-principles study of electronic and magnetic structure of $\alpha-\mathrm{Pu}, \delta$-Pu, americium, and curium, J. Magn. Magn. Mater. 272-276, E329 (2004).

[9] P. Söderlind and B. Sadigh, Density-Functional Calculations of $\alpha, \beta, \gamma, \delta, \delta^{\prime}$, and $\epsilon$ Plutonium, Phys. Rev. Lett. 92, 185702 (2004).
[10] P. Söderlind, G. Kotliar, K. Haule, P. M. Oppeneer, and D. Guillaumont, Computational modeling of actinide materials and complexes, MRS Bull. 35, 883 (2010).

[11] A. Dewaele, J. Bouchet, F. Occelli, M. Hanfland, and G. Garbarino, Refinement of the equation of state of $\alpha$-uranium, Phys. Rev. B 88, 134202 (2013).

[12] P. Söderlind, B. Johansson, and O. Eriksson, Theoretical zerotemperature phase diagram for neptunium metal, Phys. Rev. B 52, 1631 (1995).

[13] P. Faure and C. Genestier, X-ray diffraction study of pure plutonium under pressure, J. Nucl. Mater. 385, 38 (2009).

[14] H. Ledbetter, A. Migliori, J. Betts, S. Harrington, and S. ElKhatib, Zero-temperature bulk modulus of alpha-plutonium, Phys. Rev. B 71, 172101 (2005).

[15] P. Söderlind, A. Landa, J. E. Klepeis, Y. Suzuki, and A. Migliori, Elastic properties of $\mathrm{Pu}$ metal and Pu-Ga alloys, Phys. Rev. B 81, 224110 (2010).

[16] S. Heathman, R. G. Haire, T. Le Bihan, A. Lindbaum, K. Litfin, Y. Méresse, and H. Libotte, Pressure Induces Major Changes in the Nature of Americium's $5 f$ Electrons, Phys. Rev. Lett. 85, 2961 (2000).

[17] R. Haire, J. Peterson, U. Benedict, C. Dufour, and J. Itié, X-ray diffraction of curium-248 metal under pressures of up to $52 \mathrm{GPa}$, J. Less-Common Met. 109, 71 (1985).

[18] S. Heathman, R. G. Haire, T. Le Bihan, A. Lindbaum, M. Idiri, P. Normile, S. Li, R. Ahuja, B. Johansson, and G. H. Lander, A high-pressure structure in curium linked to magnetism, Science 309, 110 (2005).

[19] O. J. Wick, Plutonium Handbook A Guide to the Technology (Gordon and Breach, New York, 1967). 
[20] W. H. Zachariasen, Crystal chemical studies of the $5 f$-series of elements. XVII. The crystal structure of neptunium metal, Acta Crystallogr. 5, 660 (1952).

[21] S. Dabos, C. Dufour, U. Benedict, and M. Pagès, Bulk modulus and P-V relationship up to $52 \mathrm{GPa}$ of neptunium metal at room temperature, J. Magn. Magn. Mater. 63, 661 (1987).

[22] D. C. Wallace, Electronic and phonon properties of six crystalline phases of Pu metal, Phys. Rev. B 58, 15433 (1998).

[23] M. D. Jones, J. C. Boettger, R. C. Albers, and D. J. Singh, Theoretical atomic volumes of the light actinides, Phys. Rev. B 61, 4644 (2000).

[24] S. Y. Savrasov, G. Kotliar, and E. Abrahams, Correlated electrons in delta-plutonium within a dynamical mean-field picture, Nature (London) 410, 793 (2001).

[25] S. Y. Savrasov, K. Haule, and G. Kotliar, Many-Body Electronic Structure of Americium Metal, Phys. Rev. Lett. 96, 036404 (2006).
[26] Y. Baer and J. K. Lang, High-energy spectroscopic study of the occupied and unoccupied $5 f$ and valence states in Th and $\mathrm{U}$ metals, Phys. Rev. B 21, 2060 (1980).

[27] J. Naegele, L. Cox, and J. Ward, Photoelectron spectroscopy (UPS/XPS) study of $\mathrm{Np}_{2} \mathrm{O}_{3}$ formation on the surface of neptunium metal, Inorganica Chimica Acta 139, 327 (1987).

[28] T. Gouder, L. Havela, F. Wastin, and J. Rebizant, Evidence for the $5 f$ localisation in thin Pu layers, Europhys. Lett. 55, 705 (2001).

[29] J. R. Naegele, L. Manes, J. C. Spirlet, and W. Müller, Localization of $5 f$ Electrons in Americium: A Photoemission Study, Phys. Rev. Lett. 52, 1834 (1984).

[30] T. Gouder, G. van der Laan, A. B. Shick, R. G. Haire, and R. Caciuffo, Electronic structure of elemental curium studied by photoemission, Phys. Rev. B 83, 125111 (2011). 